\title{
Surgery versus Radiofrequency Ablation for Small Hepatocellular Carcinoma: A Randomized Controlled Trial (SURF Trial)
}

\author{
Tadatoshi Takayama $^{a}$ Kiyoshi Hasegawa ${ }^{b}$ Namiki Izumic Masatoshi Kudo ${ }^{d}$ \\ Mitsuo Shimada ${ }^{e}$ Naoki Yamanaka ${ }^{f}$ Masafumi Inomatag ${ }^{g}$ Shuichi Kaneko $^{\text {h }}$ \\ Hisashi Nakayama ${ }^{a}$ Yoshikuni Kawaguchi ${ }^{b}$ Kosuke Kashiwabara ${ }^{i}$ Ryosuke Tateishi ${ }^{j}$

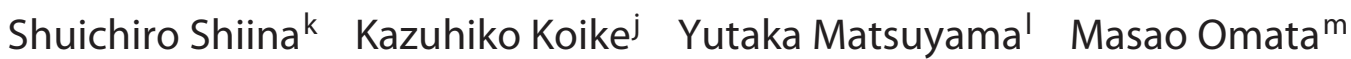 \\ Masatoshi Makuuchin ${ }^{n}$ Norihiro Kokudo $^{\circ}$

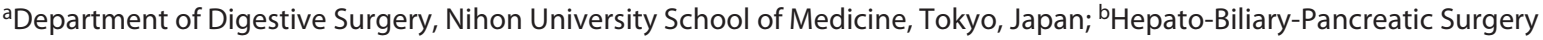
Division, Department of Surgery, Graduate School of Medicine, the University of Tokyo, Tokyo, Japan; 'Department of Gastroenterology, Musashino Red Cross Hospital, Tokyo, Japan; ' Department of Gastroenterology and Hepatology, Kinki University School of Medicine, Osaka, Japan; 'Department of Surgery, Tokushima University School of Medicine, Tokushima, Japan; ${ }^{f}$ Meiwa Hospital, Hyogo, Japan; '9Department of Gastroenterological and Pediatric Surgery, Oita University Faculty of Medicine, Oita, Japan; hDepartment of Gastroenterology, Graduate School of Medicine, Kanazawa University, Ishikawa, Japan; 'Biostatistics Division, Central Coordinating Unit, Clinical Research Support Center, the University of Tokyo Hospital, Tokyo, Japan; 'Department of Gastroenterology, Graduate School of Medicine, the University of Tokyo, Tokyo, Japan; ' Department of Gastroenterological Imaging and Interventional Oncology, Juntendo University, Tokyo, Japan; 'Department of Biostatistics, School of Public Health, the University of Tokyo, Tokyo, Japan; mInternal Medicine, Yamanashi Prefectural Central Hospital, Yamanashi, Japan; ${ }^{n}$ Koto Hospital, Tokyo, Japan; ${ }^{\circ}$ National Center for
\end{abstract} \\ Global Health and Medicine, Tokyo, Japan
}

\section{Keywords}

Hepatocellular carcinoma · Liver resection · Hepatectomy · Ablation · Randomized controlled trial

\begin{abstract}
Introduction: It remains unclear which surgery or radiofrequency ablation (RFA) is the more effective treatment for small hepatocellular carcinoma (HCC). We aimed to compare survival between patients undergoing surgery (surgery group) and patients undergoing RFA (RFA group). Methods: We conducted a randomized controlled trial involving 49 institutions in Japan. Patients with Child-Pugh scores $\leq 7$, largest HCC diameter $\leq 3 \mathrm{~cm}$, and $\leq 3 \mathrm{HCC}$ nodules were consid-
\end{abstract}

ered eligible. The co-primary endpoints were recurrencefree survival (RFS) and overall survival (OS). The current study reports the final result of RFS, and the follow-up of OS is still ongoing. Results: During 2009-2015, 308 patients were registered. After excluding ineligible patients, the surgery and RFA groups included 150 and 151 patients, respectively. Baseline factors did not differ significantly between the groups. In both groups, $90 \%$ of patients had solitary HCC. The median largest HCC diameter was $1.8 \mathrm{~cm}$ (interquartile range $[I Q R], 1.5-2.2 \mathrm{~cm})$ in the surgery group and $1.8 \mathrm{~cm}(I Q R$, $1.5-2.3 \mathrm{~cm}$ ) in the RFA group. The median procedure duration ( 274 vs. 40 min, $p<0.01$ ) and the median duration of hospital stay ( 17 days vs. 10 days, $p<0.01$ ) were longer in the surgery group than in the RFA group. RFS did not differ sig-
(C) 2021 The Author(s)

Published by S. Karger AG, Basel

This is an Open Access article licensed under the Creative Commons Attribution-NonCommercial-4.0 International License (CC BY-NC) (http://www.karger.com/Services/OpenAccessLicense), applicable to the online version of the article only. Usage and distribution for commercial purposes requires written permission.
Correspondence to:

Kiyoshi Hasegawa, kihase-tky@umin.ac.jp 
nificantly between the groups as the median RFS was 3.5 (95\% confidence interval $[\mathrm{Cl}], 2.6-5.1$ ) years in the surgery group and $3.0(95 \% \mathrm{Cl}, 2.4-5.6)$ years in the RFA group (hazard ratio, $0.92 ; 95 \% \mathrm{Cl}, 0.67-1.25 ; p=0.58$ ). Discussion/Conclusion: Our study did not show which surgery or RFA is the better treatment option for small HCC.

(C) 2021 The Author(s)

Published by S. Karger AG, Basel

\section{Introduction}

Hepatocellular carcinoma (HCC) is the 6th most common cancer worldwide and the 4th leading cause of cancer-related deaths in 2018 [1]. The Barcelona Clinic Liver Cancer staging classification recommends surgery only for patients with stage 0 disease [2] (solitary HCC diameter $<2 \mathrm{~cm}$ and Child-Pugh score A) and with stage A (solitary HCC) and radiofrequency ablation (RFA) for stage 0 and stage A patients (patients with the largest HCC diameter $\leq 3 \mathrm{~cm}$ and $\leq 3$ nodules) [3]. The Japanese clinical practice guidelines for HCC (4th JSH-HCC guidelines) recommends both surgery and RFA for patients with the largest $\mathrm{HCC}$ diameter $\leq 3 \mathrm{~cm}, \mathrm{HCC} \leq 3$ nodules, and Child-Pugh grade A or B [4]. Over time, advancements in surgery have made safe limited and anatomic resection of Couinaud's segments possible [5]. Similarly, advancements in electrocoagulation have improved the efficacy of RFA as a curative-intent treatment option [6].

Four relevant randomized controlled trials (RCTs) have heretofore been conducted [7-10]. Of the 4 RCTs, one showed that surgery was associated with better survival than RFA, whereas the other three did not show significant differences in survival between patients undergoing surgery and patients undergoing RFA. The 4 RCTs had had the following shortcomings: lack of clear hypothesis, insufficient patient' follow-up, and unknown treatment allocation. To answer this important clinical question, we conducted a multicenter RCT to compare outcomes of patients undergoing surgery and patients undergoing RFA for small HCC.

\section{Materials and Methods}

\section{Study Population}

The subjects consisted of patients with the largest HCC diameter $\leq 3 \mathrm{~cm}$ and $\leq 3$ HCC nodules. Both liver surgeons and gastroenterologists reviewed radiographic images of all the patients and confirmed that they were treatable using both surgery and RFA. We included patients who had undergone trans-arterial chemoembolization (TACE) for the same target lesions within 3 months before the treatment protocol because sequential treatment (i.e.,
TACE to surgery or RFA) is commonly performed in clinical practice on the basis of data supporting lower recurrence after the sequential treatment [11]. The inclusion criteria were as follows: age of 20-80 years, Eastern Cooperative Oncology Group [12] performance status of $0-2$, Child-Pugh score $\leq 7$, the absence of extrahepatic metastasis, the absence of macrovascular invasion, white blood cell count of $2,000-10,000 / \mathrm{mm}^{3}$, platelet count $\geq 50,000$ / $\mathrm{mm}^{3}$, hemoglobin level $\geq 8.0 \mathrm{~g} / \mathrm{dL}$, serum total bilirubin level $\leq 2.0$ $\mathrm{mg} / \mathrm{dL}$, prothrombin time $\geq 50 \%$, serum creatinine level $\leq 1.5 \mathrm{mg} /$ $\mathrm{dL}$, and blood urea nitrogen level $\leq 35 \mathrm{mg} / \mathrm{dL}$. The exclusion criteria were as follows: concurrent cancer in other organs, history of different cancer within 5 years before enrollment, poor cardiac function, severe respiratory symptoms, contrast media hypersensitivity reactions, renal dysfunction, mental illness, and pregnancy. Our protocol did not mandate to report other parameters associated with liver function including indocyanine green retention rate, the Model of End-stage Liver Disease score, and the presence/ absence of portal hypertension. This study was approved by the institutional Ethics Committees of the participating institutions and was conducted in accordance with good clinical practice guidelines and the Helsinki Declaration. Our study was registered in the UMIN-CTR (UMIN000001795, https://center.umin.ac.jp/ ctr/index.htm). All the patients provided written informed consent. All the authors had access to the study data and had reviewed and approved the final manuscript.

\section{Radiographic Definition of HCC}

HCC was defined radiographically as lesions $(\geq 1 \mathrm{~cm})$ that show high enhancement in the early phase and washout in the late phase of the dynamic computed tomography (CT) study with contrast enhancement [4]. The HCC diameter was measured by either the early or late phase. Tumor biopsy was not required for the diagnosis.

\section{Multidisciplinary Discussion before Enrollment}

Before enrollment, the liver surgeons and gastroenterologists who performed RFA carefully assessed and reviewed the HCC location and diameter in all the study participants. The study group confirmed that all the patients can be treated using both surgery and RFA. Given the variety of clinical practice in each institution, the criteria for feasibility of both treatments were not specified in detail.

\section{Study Design}

The multi-institutional RCT was conducted in 118 institutions in Japan. All eligible patients were randomly allocated (1:1) to either the surgery group or the RFA group using the probabilistic minimization method. For each eligible patient with informed consent, registration form was sent to the data center by FAX, and data center staffs registered the patient in the electronic data capturing system. The minimization algorithm was automatically run in the electronic data capturing system, and the resulted treatment allocation was informed to the institution. The adjusted factors in minimization were age ( $<60$ years or $\geq 60$ years), hepatitis $C$ virus infection, number of tumors (solitary or multiple), tumor diameter $(\leq 2 \mathrm{~cm}$ or $>2 \mathrm{~cm})$, and institutions.

Surgery and RFA for HCC were both performed after preoperative evaluation of liver function and pretreatment according to decisions at each institution. Technical details of both surgery and RFA were not specified in the protocol. Surgery was performed using ei- 


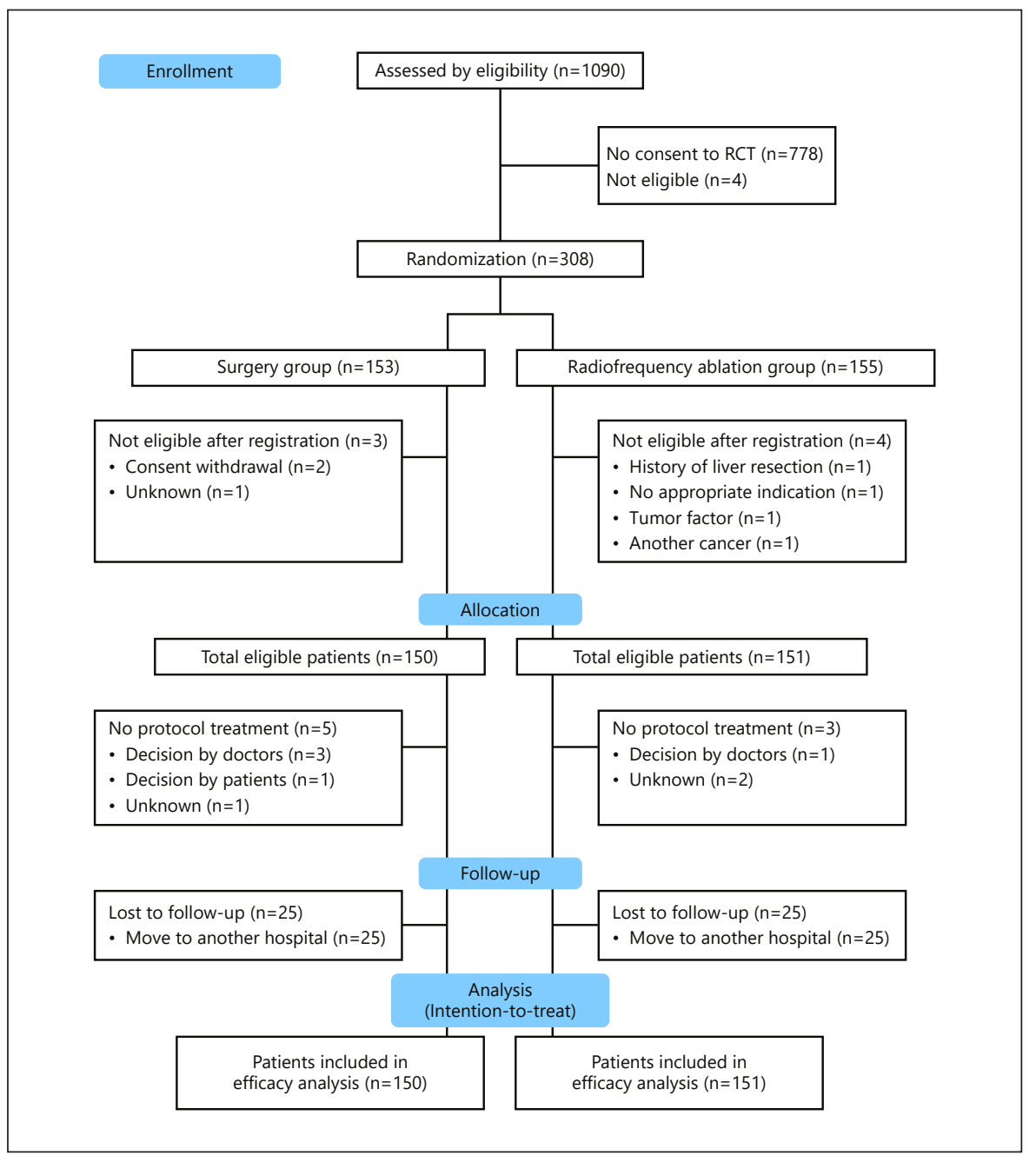

Fig. 1. Patient flow chart.

ther an open or laparoscopic approach. RFA was percutaneously performed under the guidance of ultrasonography so that unstained, low-density areas appear widely along the entire margin after RFA than low-density areas in the late phase of CT observed before RFA. Additional RFAs performed within 2 weeks after the initial RFA to lesions which had evidence of incomplete treatment by CT imaging were considered to be part of the 1 series of treatment. RFA was deemed successful when the tumor necrotic effect reaches TE4a (complete response with enough ablative margin) according to the Response Evaluation Criteria in Cancer of the Liver [13]. TE4b (complete response without enough ablative margin) was also considered a successful treatment effect. Adverse effects after treatments were collected but posttreatment complications were not.

\section{Endpoints}

The co-primary endpoints were recurrence-free survival (RFS) and overall survival (OS). OS and RFS were defined as the duration from the date of enrollment to the date of death of any cause and the date of the diagnosis of recurrence, respectively. Recurrence or primary disease-related death without recurrence was defined as an event for RFS analysis. Local recurrence was defined as follows: (1) appearance of typical HCC adjacent to the treated area; (2) ap- pearance of portal vein tumor thrombus in an area including the treated area; and (3) appearance of a tumor adjacent to the treated area and an increase in liver cancer-related tumor markers again. Patients who died of other diseases without recurrence were censored at the time of death. At 3 years after the last accrual of patients, a final analysis of RFS and an interim analysis of OS were planned because the efficacy of local control (i.e., local recurrence) is a matter of interest for selecting surgery or RFA. A final analysis of OS was planned at 5 years after the last accrual of patients. The follow-up of OS is still ongoing.

Follow-Up

Posttreatment evaluations were performed once every 2 months from the date of enrollment to 3 years after enrollment and once every 4 months from 3 years after enrollment to the end of this study (i.e., 5 years after the last enrollment). Dynamic abdominal CT study with contrast enhancement and chest X-ray were performed once every 4 months. If a patient develops contrast media hypersensitivity reactions during follow-up, dynamic magnetic resonance imaging study with gadolinium or gadolinium-ethoxybenzyl-diethylenetriamine penta-acetic acid, instead of CT, is used for follow-up assessment. 
Table 1. Demographic and clinicopathologic characteristics of the surgery and RFA groups

\begin{tabular}{|c|c|c|c|}
\hline Characteristics & Surgery group $(n=150)$ & RFA group $(n=151)$ & $p$ value \\
\hline \multicolumn{4}{|l|}{ Patient factors } \\
\hline Age, median (IQR), years & $68(63-74)$ & $69(63-74)$ & 0.85 \\
\hline Sex, male:female & $112: 38$ & $108: 43$ & 0.54 \\
\hline \multicolumn{4}{|l|}{ Performance status, $n(\%)$} \\
\hline 0 & $147(98)$ & $146(97)$ & \multirow{3}{*}{0.56} \\
\hline 1 & $3(2)$ & $4(3)$ & \\
\hline 2 & 0 & $1(1)$ & \\
\hline \multicolumn{4}{|l|}{ Child-Pugh score, $n$ (\%) } \\
\hline 5 & $117(78)$ & $129(85)$ & \multirow{3}{*}{0.07} \\
\hline 6 & $22(15)$ & $20(13)$ & \\
\hline 7 & $10(7)$ & $2(1)$ & \\
\hline Hepatitis C virus infection, $n(\%)$ & $97(65)$ & $94(62)$ & 0.66 \\
\hline Hepatitis B virus infection,* $n(\%)$ & $27(18)$ & $33(22)$ & 0.42 \\
\hline Use of antiviral therapy, $n(\%)$ & $23(15)$ & $21(14)$ & 0.58 \\
\hline Serum albumin level, median (IQR), g/dL & $4.0(3.7-4.3)$ & $4.1(3.7-4.4)$ & 0.48 \\
\hline Total bilirubin level, median (IQR), mg/dL & $0.7(0.5-1.0)$ & $0.8(0.6-1.0)$ & 0.58 \\
\hline Prothrombin time, median (IQR), \% & $91(81-99)$ & $94(86-100)$ & 0.01 \\
\hline Platelet count, median (IQR), $10^{4} / \mu \mathrm{L}$ & $13.0(9.9-16.8)$ & $13.5(9.8-17.5)$ & 0.62 \\
\hline Creatinine level, median (IQR), mg/dL & $0.7(0.6-0.9)$ & $0.7(0.6-0.9)$ & 0.73 \\
\hline Absence of ascites, ${ }^{*} n(\%)$ & $145(97)$ & $149(99)$ & 0.16 \\
\hline History of other cancers, ${ }^{*} n(\%)$ & $13(9)$ & $7(5)$ & 0.23 \\
\hline Comorbidity, ischemic heart disease, $n(\%)$ & $2(1)$ & $2(1)$ & 0.61 \\
\hline Pretreatment TACE* & $11(7)$ & $12(8)$ & 0.59 \\
\hline \multicolumn{4}{|l|}{ HCC clinical factors } \\
\hline $\mathrm{HCCs}, n$ & $1(1-1)$ & $1(1-1)$ & 0.97 \\
\hline$\geq 2, n(\%)$ & $15(10)$ & $15(10)$ & 0.98 \\
\hline Largest HCC diameter, median (IQR), cm & $1.8(1.5-2.2)$ & $1.8(1.5-2.3)$ & 0.89 \\
\hline$\leq 2.0 \mathrm{~cm}, n(\%)$ & $98(65)$ & $101(67)$ & 0.78 \\
\hline \multicolumn{4}{|l|}{ HCC histopathologic factors, $n(\%)$} \\
\hline $\mathrm{HCC}$ & $131(87)$ & $30(20)$ & \multirow[t]{5}{*}{-} \\
\hline Well-differentiated & $39(26)$ & $10(7)$ & \\
\hline Moderately differentiated & $85(57)$ & $16(11)$ & \\
\hline Poorly differentiated & $7(5)$ & $4(3)$ & \\
\hline Unknown & $19(13)$ & $121(80)$ & \\
\hline
\end{tabular}

* Data missing on hepatitis B virus infection for 1 patient, the absence of ascites for 1 patient, history of other cancers for 1 patient, comorbidity of ischemic heart disease for 1 patient, and pretreatment TACE for 1 patient. ${ }^{\dagger}$ Including patients treated with diuretics.

\section{Sample Size}

The 3-year RFS rates in patients who underwent initial treatment for HCC in the University of Tokyo Hospital before the start of this study were $43.3 \%$ in patients undergoing surgery and $32.5 \%$ in patients undergoing RFA. The 5-year OS rates were $67.4 \%$ in patients undergoing surgery and $70.3 \%$ in patients undergoing RFA. Under the assumption that 3-year RFS rates were $45 \%$ in patients undergoing surgery and $35 \%$ in patients undergoing RFA, the number of subjects needed for statistical significance was 285 per group (with $\alpha=0.05$, power of $80 \%, 2$-sided, enrollment period of 3 years, and follow-up period of 6 years). Under the assumption that 3 -year RFS rates were $45 \%$ in patients undergoing surgery and $30 \%$ in patients undergoing RFA (intergroup difference of $15 \%), 123$ patients per group were needed. For a minimum of $10 \%$ difference between patients undergoing surgery and patients undergoing RFA, we planned to enroll 300 patients per group.

\section{Statistical Analysis}

Categorical variables were expressed as numbers and percentages and compared using Fisher's exact test or $\chi^{2}$ test. Continuous variables were expressed as median values with interquartile ranges (IQRs) and compared using Wilcoxon's rank-sum test. Primary endpoint was assessed based on intention-to-treat analysis. RFS curves were constructed using the Kaplan-Meier method and compared using the log-rank test stratified by the allocation factors except institutions. A Cox proportional hazards model analysis was performed to assess hazard ratios (HRs) and 95\% confidence 
Table 2. Intra-procedure and post-procedure outcomes in the surgery and RFA groups

\begin{tabular}{lllc}
\hline & Surgery group $(n=150)$ & RFA group $(n=151)$ & $p$ value \\
\hline Procedure duration, median (IQR), min & $274(203-341)$ & $40(24-70)$ & $<0.01$ \\
Blood loss, median (IQR), mL & $285(103-635)$ & - & - \\
Duration of hospital stay, median (IQR), days & $17(12-23)$ & $10(7-16)$ & $<0.01$ \\
Adverse events, $n$ (\%) & $5(3)$ & 0 & - \\
$\quad$ Ascites & $3(2)$ & 0 & - \\
$\quad$ Bile leakage & $1(1)$ & 0 & - \\
Abscess & $1(1)$ & 0 & - \\
$\quad$ Mortality & 0 & 0 & - \\
\hline
\end{tabular}

intervals (CIs) for each factor after confirming intergroup proportional hazards. The CIs of the 3-year and 5-year RFS of each treatment group were calculated using Greenwood's formula. $p<0.05$ was considered statistically significant. Statistical analysis was performed using the SAS software (SAS Institute, Cary, NC, USA).

\section{Results}

\section{Study Population}

From April 1, 2009, to August 31, 2015, 1,090 patients from 49 institutions met the inclusion criteria, and 308 patients (28.3\%) were randomized including 197 patients accrued from 9 institutions and 111 patients accrued in the other 40 institutions (Fig. 1). Of the 308 patients, 153 and 155 were randomly assigned to undergo surgery and RFA, respectively. After excluding 7 ineligible patients, 150 patients were eligible for surgery, and 151 were eligible for RFA. In March 2015, an independent data monitoring committee recommended that the study group stop patient accrual without reaching the targeted number of patients $(n=600)$ because the target number of patients was most likely unachievable on the basis of the low speed of accrual. Therefore, the 150 patients undergoing surgery (the surgery group) including 5 patients who did not undergo protocol treatment and 9 patients (6\%) with lost to follow-up within 3 years from randomization and the 151 patients undergoing RFA (the RFA group) including 3 patients who did not undergo protocol treatment and 6 patients (4\%) with lost to follow-up within 3 years from randomization were treated in an intention-to-treat fashion (Fig. 1). No significant differences were observed in demographic and clinicopathologic characteristics between the 2 groups (Table 1). In both groups, $90 \%$ of patients had solitary HCC, and the median largest HCC diameter was $1.8 \mathrm{~cm}$. In the surgery group, 39 patients $(26.0 \%)$ had well-differentiated HCC,
$85(56.7 \%)$ had moderately differentiated HCC, and 7 (4.7\%) had poorly differentiated HCC. In the RFA group, there was no data on 121 patients $(80.1 \%)$ regarding HCC differentiation.

\section{Intra-Procedure and Post-Procedure Outcomes}

Table 2 shows the intra-procedure and post-procedure outcomes. The median (IQR) procedure duration was significantly longer in the surgery group than in the RFA group: 274 (203-341) minutes versus 40 (24-70) minutes. Surgery was performed using anatomic liver resection in 69 patients (46\%) and nonanatomic resection in 57 patients (38\%) with 24 patients (16\%) having missing information. Additional ablation within 2 weeks after the initial RFA was performed in 17 patients (11\%) including 14 patients (9\%) undergoing one additional RFA, 2 patients (1\%) undergoing two additional RFA, and a patient (1\%) undergoing three additional RFA and not in 121 patients $(80 \%)$. The median (IQR) duration of the hospital stay was significantly longer in the surgery group than in the RFA group: 17 (12-23) days versus 10 (7-16) days. There was no mortality in any of the groups. In the surgery group, the following adverse events developed in 5 patients (3\%): ascites $(n=3,2 \%)$, bile leakage $(n=1,1 \%)$, and abscess $(n$ $=1,1 \%)$. Adverse events did not develop in any patient in the RFA group according to the case report forms.

\section{RFS}

The median (range) post-enrollment follow-up period was $5.04(0.36-9.49)$ years in the surgery group and 4.99 $(0.00-8.70)$ years in the RFA group. The median RFS did not differ significantly between the surgery and the RFA groups: 3.46 years vs. 3.04 years; HR, 0.92; 95\% CI, 0.67 $1.25 ; p=0.58$ ) (Fig. 2). In the surgery group, the 3 -year RFS and 5-year RFS were 54.6\% (95\% CI, 45.8\%-62.6\%) and $42.7 \%$ (95\% CI, 33.8\%-51.3\%), respectively. In the RFA group, the 3-year RFS and 5-year RFS were 50.5\% 
Fig. 2. RFS between the surgery and RFA groups.

Table 3. First recurrence and treatments in the surgery and RFA groups

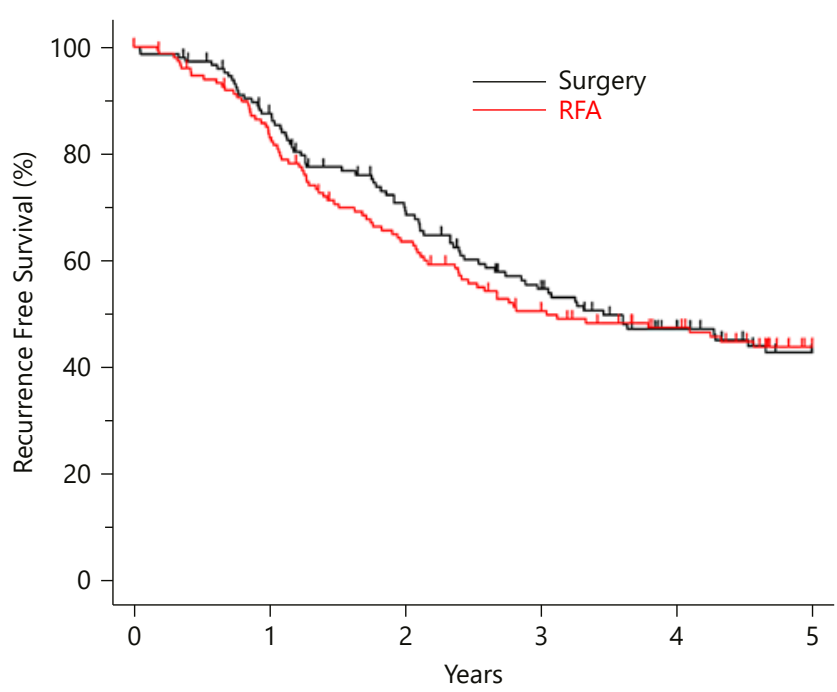

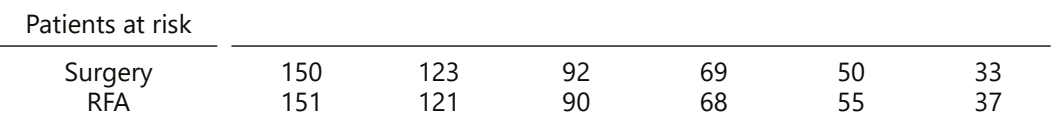

\begin{tabular}{|c|c|c|c|}
\hline Characteristics & Surgery group $(n=82)$ & RFA group $(n=86)$ & $p$ value \\
\hline \multicolumn{4}{|c|}{ Site of recurrence, $n$ (\%) } \\
\hline Liver* & $80(98)$ & $85(99)$ & $>0.99$ \\
\hline Extrahepatic** & $1(1)$ & $1(1)$ & $>0.99$ \\
\hline Bone & 0 & $1(1)$ & $>0.99$ \\
\hline Lymph node & $1(1)$ & $1(1)$ & $>0.99$ \\
\hline Others & $1(1)$ & 0 & 0.98 \\
\hline \multicolumn{4}{|c|}{ Number of recurrences in the liver, $n$ (\%) } \\
\hline Single & $49(60 \%)$ & $61(71 \%)$ & \multirow{2}{*}{0.21} \\
\hline Multiple & $31(38 \%)$ & $24(28 \%)$ & \\
\hline \multicolumn{4}{|c|}{ Local recurrence, ${ }^{\# \#} n$ (\%) } \\
\hline Absent & $66(80)$ & $61(71)$ & \multirow{2}{*}{0.07} \\
\hline Present & $12(15)$ & $24(28)$ & \\
\hline \multicolumn{4}{|c|}{ Vascular invasion, $^{\dagger} n(\%)$} \\
\hline Absent & $77(94)$ & $81(94)$ & \multirow{2}{*}{0.42} \\
\hline Present & $1(1)$ & $4(5)$ & \\
\hline \multicolumn{4}{|c|}{ Treatments for first recurrence, $n$ (\%) } \\
\hline Surgery & $13(16)$ & $7(8)$ & 0.19 \\
\hline RFA & $48(59)$ & $52(60)$ & 0.92 \\
\hline TACE & $24(29)$ & $30(35)$ & 0.54 \\
\hline Others & $2(2)$ & $3(3)$ & $>0.99$ \\
\hline
\end{tabular}

* Liver metastasis was missed in 2 patients in the surgery group. ** Extrahepatic recurrence was missed in 2 patients in the surgery group and in 1 patient in the RFA group. "Liver recurrence was missed in 2 patients in the surgery group and in 1 patient in the RFA group. ${ }^{\#}$ Local recurrence was missed in 4 patients in the surgery group and in 1 patient in the RFA group. ${ }^{\dagger}$ Vascular invasion was missed in 4 patients in the surgery group and in 1 patient in the RFA group. ${ }^{\ddagger} X^{2}$ test for categorical variables and Wilcoxon's rank-sum test for continuous variables. 


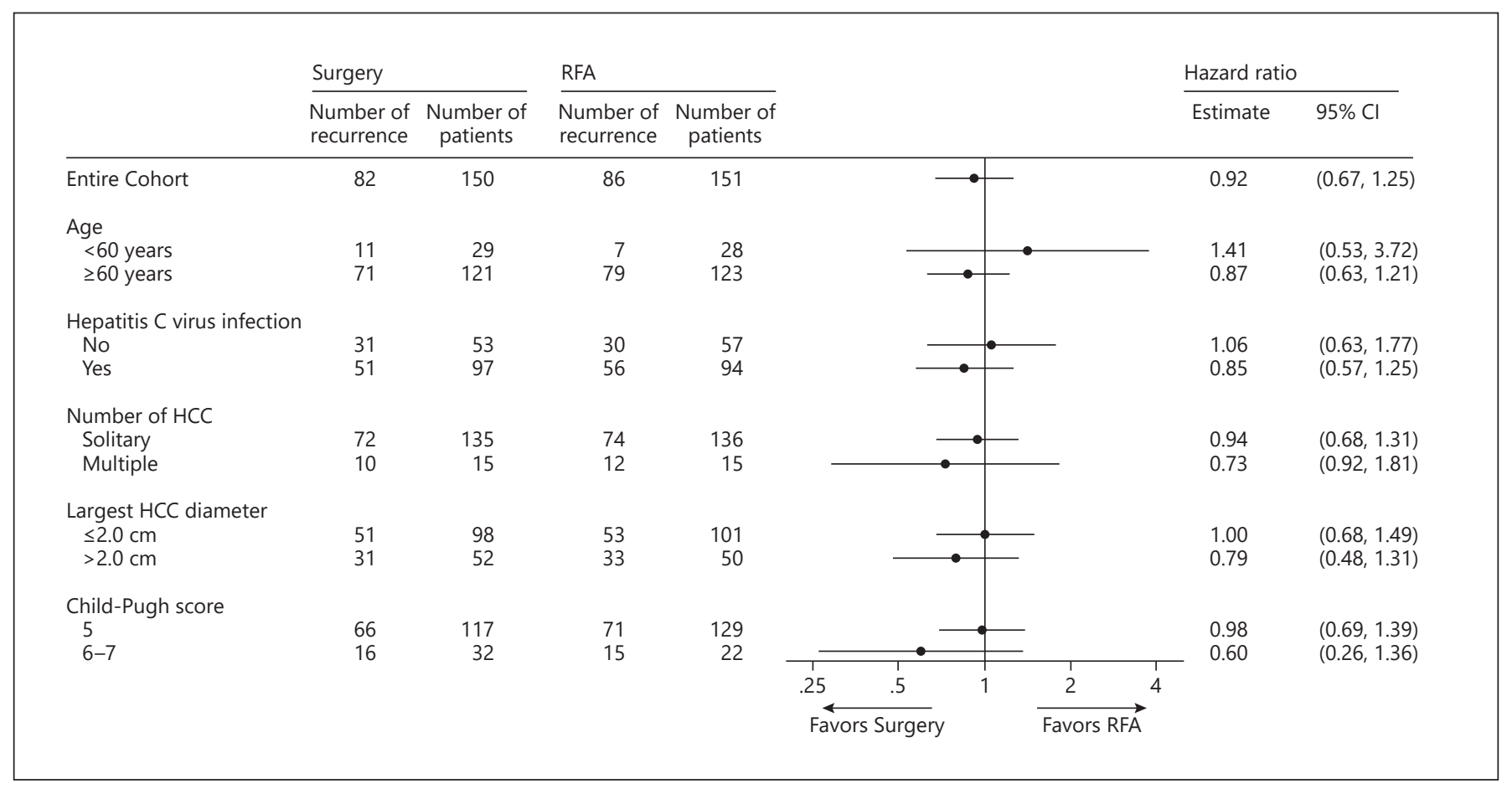

Fig. 3. Subgroup analysis of RFS in the surgery and RFA groups.

(95\% CI, $42.0 \%-58.4 \%)$ and $43.8 \%$ (95\% CI, 35.3\%$51.9 \%)$, respectively.

In patients who did not undergo TACE, the median RFS did not differ significantly between the surgery group $(n=139)$ and the RFA group $(n=138): 3.31$ years vs. 2.82 years (HR, 0.90 ; 95\% CI, $0.65-1.25$; $p=0.52$ ) (online suppl. Fig. 1; for all online suppl. material, see www.karger. com/doi/10.1159/000521665).

\section{First Recurrence after Procedures}

Recurrences were diagnosed in 82 patients (54.7\%) in the surgery group and in 86 patients $(57.0 \%)$ in the RFA group (Table 3 ). In both groups, most recurrences were found in the liver of 80 patients (53.3\%) in the surgery group and 86 patients (57.0\%) in the RFA group. Extrahepatic recurrence was found in 2 patients in each group. The local recurrence rate was lower in the surgery group than in the RFA group: $15.0 \%$ versus $28.0 \%, p=0.07$. The type of treatment for recurrence did not differ significantly between the groups. Of the 82 patients in the surgery group who had recurrences, 13 (15.9\%) underwent surgery, 48 (58.5\%) underwent RFA, and 24 (29.3\%) underwent TACE. Of the 86 patients in the RFA group who had recurrences, 7 (8.1\%) underwent surgery, 52 (60.5\%) underwent RFA, and 31 (36.0\%) underwent TACE.

Surgery versus Radiofrequency Ablation

for Hepatocellular Carcinoma

\section{Subgroup Analysis}

Figure 3 shows the subgroup analysis, which was stratified by age ( $<60$ years vs. $\geq 60$ years), hepatitis $C$ virus infection status, number of HCC nodules (solitary vs. multiple), largest HCC diameter ( $\leq 2.0 \mathrm{~cm}$ vs. $>2.0 \mathrm{~cm}$ ), and Child-Pugh score (5 vs. 6-7). RFS did not differ significantly between the surgery and RFA groups in each subgroup.

\section{Discussion/Conclusion}

In patients with the largest HCC diameter $\leq 3 \mathrm{~cm}$ and $\leq 3$ HCC nodules, our trial did not find significant differences in RFS between the surgery and RFA groups. The median RFS was 3.5 years in the surgery group and 3.0 years in the RFA group. According to our protocol, assessment of OS is scheduled after August 2020.

Our study is the 5th RCT to compare survival between surgery and RFA groups. Compared to the 4 previous RCTs of HCC treatment with surgery or RFA [7-10], our RCT included the highest number of patients who were mostly associated with hepatitis C. Three previous RCTs did not find surgery superior to RFA in terms of patient OS [7-9]. However, RFS was significantly longer $[8,10]$ 
or tended to be longer $[7,9]$ in patients undergoing surgery than in patients undergoing RFA. Our study did not find significant differences in survival between the surgery and RFA groups. This discrepancy can be attributed to the reasons presented below. First, the tumor statuses which influences prognosis after treatments [14] were different (Millan criteria $[8,10]$, solitary tumor of diameter $<5 \mathrm{~cm}$, up to 2 tumors $<4 \mathrm{~cm}$ in diameter [9], and up to 3 tumors $\leq 3 \mathrm{~cm}$ in diameter as in our study), and this indicates that the relatively small tumor size in our study is a limitation. Consequently, the advantage of surgery in this series may be minimal. Second, all the previous RCTs were based on a single-center trial, and the operative procedures (anatomical resection [7, 8] or nonanatomical resection with surgical margin $>1 \mathrm{~cm}[9,10])$ and RFA could be integrated. Our study is a multi-institutional joint research involving 49 institutions; therefore, the curability of HCC using surgery seemed variable. Third, the 4 RCTs were conducted in China, and they indicate that most causes of hepatocarcinogenesis were associated with hepatitis B viral infection. However, in our study, more than $60 \%$ of the patients had hepatitis $C$ viral infection. Therefore, based on the multicentric origin, recurrence may be more frequent in Japan than in China. The ratio of local recurrence to second primary lesions after surgery and RFA may be smaller than that reported in previous studies.

Two types of recurrence should be considered when addressing recurrence after local therapy of HCC. The first type is recurrence near treated areas. HCC is reported to advance into the portal vein, and recurrence may occur in the same Couinaud segment as the treated HCC [15]. Two previous meta-analyses reported that the local recurrence rate was significantly higher in patients undergoing RFA than in patients undergoing surgery $[16$, 17], and this is consistent with our study. This indicates that surgery may be associated with low local recurrence as surgery can remove HCC and surrounding liver parenchyma [18]. RFA has advantages over surgery with regard to procedure duration and duration of the hospital stay. The second type is de novo intrahepatic recurrence (i.e., multicentric HCC). This type of recurrence accounts for $30-40 \%$ of recurrences after HCC treatments $[19,20]$. The second type of recurrence limit efforts aimed at addressing treatment efficacy between surgery and RFA. Analysis of RFS includes both types of recurrence; however, the second type of recurrence is likely not related to the type of local therapy but to background liver function.

Our study should be understood in the context of the limitations. First, the target number of patient was not reached because the speed of patient accrual was low. This may further decrease the number of patients for subgroup analysis. Nevertheless, as the RFS curves of both groups overlapped, we assumed that the result may have been the same if we registered the target number of patients. Using the current data, we calculated conditional power (i.e., the probability that statistically significant RFS results would have been observed if a total of 570 patients which is the initially planned number of patients had been enrolled and if the HR was 0.90), and the calculated conditional power was less than $8 \%$. Although the current study had approximately $15 \%$ of lost-to-follow-up patients in each group, the survival outcome was investigated with the support of hospitals where the patients moved. Second, more than $90 \%$ of the patients in our study had solitary HCC nodule and approximately $65 \%$ of the patients had the largest HCC diameter $<2.0 \mathrm{~cm}$. This tumor characteristic may have influenced our study results. In fact, the 3 -year RFS rate of the surgery group in our study was comparable to the 3-year RFS rate of the RFA group. In contrast, the 3-year RFS rate of the RFA group was higher than the 3-year RFS rate of patients undergoing RFA in our retrospective cohort. We used our retrospective cohort for sample size estimation; however, compared to the RFA group of our current cohort, the patients undergoing RFA of the retrospective cohort had multiple and large HCCs. This most likely explains the difference in the 3-year RFS rate between the RFA group of our current cohort and patients undergoing RFA of our retrospective cohort. Third, the success of RFA was not clearly defined as shown by the International Working Group on ImageGuided Tumor Ablation (i.e., 5-10 mm ablation margin) [21]. Last, the primary endpoint of our study was RFS, even though the European Association for the Study of the Liver recommends time to recurrence as the primary endpoint. We reckon that this did not impact our results as there was no treatment-related death in our study, and primary disease-related death without recurrence was defined as an event for recurrence.

In conclusion, this RCT showed that, for patients with largest HCC diameter $\leq 3 \mathrm{~cm}$ and $\leq 3$ HCC nodules, RFS did not differ significantly between the surgery and RFA groups. We recommend carefulness when RFA is performed for HCCs in difficult locations because such tumors were excluded from our study and when RFA is performed for HCCs with the largest diameter $>2 \mathrm{~cm}$ as the largest HCC diameter $>2-2.5 \mathrm{~cm}$ was a risk factor for local recurrence and OS after ablation [14, 22, 23]. OS will be assessed after August 2020 as scheduled in our protocol. 


\section{Acknowledgments}

The investigators who contributed to provide relevant data for the SURF trial were listed in online supplementary Table 1 . We sincerely thank the patients for their participation in this study and their families; the data monitoring committee members, Kiwamu Okita, Takuhiro Yamaguchi, Morito Monden, Takeo Nakayama, Masatoshi Okazaki, and Tishihiko Dohzono for their support with respect to quality control of the trial; and Rei Aida, Shigeru Hayase, Yu Yamashige, Akira Yamao, Mayumi Ito, Emi Yasuda, and Yuki Nakano for their administrative supports.

\section{Statement of Ethics}

This study was approved by the institutional Ethics Committees of the participating institutions and was conducted in accordance with good clinical practice guidelines and the Helsinki Declaration. Our study was registered in the UMIN-CTR (UMIN000001795, https://center.umin.ac.jp/ctr/index.htm). All the patients provided written informed consent.

\section{Conflict of Interest Statement}

Kiyoshi Hasegawa: lecture fees from Taiho, Bayer Yakuhin, MSD K.K., and Eisai; research funding from Chugai, Otsuka, Bristol-Myers Squibb, Takeda, Taiho, Yakult, Eisai, AbbVie, Bayer Yakuhin, Kowa, Shimazu, and Nipro. Masatoshi Kudo: lecture fees from Eisai, Bayer, MDS, BMS, EA Pharma, Eli Lilly, and Chugai; research funding from Eisai, Takeda, Otsuka, Taiho, EA Pharma, Gilead Sciences, AbbVie, Sumitomo Dainippon Pharma, Chugai, and Ono Pharma; advisory role with Eisai, Ono, MSD, BMS, and Roche. Mitsuo Shimada: research funding from AbbVie, Astellas, Bayer Yakuhin, Chugai, Covidien, EA Pharma, Eisai, Novartis Pharma, Ono, Taiho, and Takeda. Yoshikuni Kawaguchi: lecture fees from Olympus, CONMED, and Otsuka. Ryosuke Tateishi: lecture fees from Chugai, Daiichi Sankyo, Eisai, GE Health Care, and
Gilead; advisory roles with AstraZeneca and Shionogi, Medtronic, MSD, Otsuka, Sumitomo Dainihon Pharma, Kowa Company, Takeda, Ono, and Shionogi. Kazuhiko Koike: research funding from MSD, AbbVie, Gilead Sciences, Otsuka, ASKA Pharma, EA Pharma, Dainippon-Sumitomo, Chugai, Eli Lilly, Taiho, Yakult, Bayer, Takeda, Tanabe-Mitsubishi, Myaln EPD, Taisho, Rohto, Eisai, and Zeria. Masatoshi Kudo is the editor-in-chief of Liver Cancer. Namiki Izumi and Norihiro Kokudo are associate editors of Liver Cancer. Kiyoshi Hasegawa is an editorial board member of Liver Cancer. None of the other authors have potential conflicts of interest to declare.

\section{Funding Sources}

This work was supported by the Japanese Foundation for Multidisciplinary Treatment of Cancer and the Health and Labor Sciences Research Grant for Clinical Cancer Research (Grant no. H21-015).

\section{Author Contributions}

Design and study conception: K.H., M.O., M.M., and N.K.; acquisition of data: T.T., K.H., N.I., M.K., M.S., N.Y., M.I., S.K., H.N., Y.K., R.T., S.S., K.K., M.O., M.M., and N.K.; analysis and interpretation: K.K., Y.M., K.H., and N.K.; participation in drafting: T.T., K.H., Y.K., and N.K.; participation in revising: all the authors. All the authors approved the final version.

\section{Data Availability Statement}

The data that support the findings of this study are not publicly available because the data availability was not mentioned in our protocol and not reviewed by the institutional Ethics Committees. However, after a relevant review of an ethical issue, the data are available from the corresponding author, K.H. upon reasonable request.

\section{References}

1 Bray F, Ferlay J, Soerjomataram I, Siegel RL, Torre LA, Jemal A. Global cancer statistics 2018: GLOBOCAN estimates of incidence and mortality worldwide for 36 cancers in 185 countries. CA Cancer J Clin. 2018 Nov;68(6): 394-424.

2 Takayama T, Makuuchi M, Hirohashi S, Sakamoto M, Yamamoto J, Shimada K, et al. Early hepatocellular carcinoma as an entity with a high rate of surgical cure. Hepatology. 1998 Nov;28(5):1241-6.

3 European Association for the Study of the Liver. Electronic address eee, European association for the study of the L. EASL clinical practice guidelines: management of hepatocellular carcinoma. J Hepatol. 2018 Jul;69(1): 182-236.

4 Kokudo N, Takemura N, Hasegawa K, Takayama T, Kubo S, Shimada M, et al. Clin-

Surgery versus Radiofrequency Ablation

for Hepatocellular Carcinoma ical practice guidelines for hepatocellular carcinoma: the Japan society of hepatology 2017 (4th JSH-HCC guidelines) 2019 update. Hepatol Res. 2019 Jul 23;49(10):1109.

5 Makuuchi M, Kosuge T, Takayama T, Yamazaki S, Kakazu T, Miyagawa S, et al. Surgery for small liver cancers. Semin Surg Oncol. 1993 Jul;9(4):298-304.

6 Bertot LC, Sato M, Tateishi R, Yoshida H, Koike K. Mortality and complication rates of percutaneous ablative techniques for the treatment of liver tumors: a systematic review. Eur Radiol. 2011 Dec;21(12):2584-96.

7 Chen MS, Li JQ, Zheng Y, Guo RP, Liang HH, Zhang YQ, et al. A prospective randomized trial comparing percutaneous local ablative therapy and partial hepatectomy for small hepatocellular carcinoma. Ann Surg. 2006 Mar; 243(3):321-8
8 Huang J, Yan L, Cheng Z, Wu H, Du L, Wang J, et al. A randomized trial comparing radiofrequency ablation and surgical resection for HCC conforming to the Milan criteria. Annals of surgery. 2010 Dec;252(6): 903-12.

9 Feng K, Yan J, Li X, Xia F, Ma K, Wang S, et al. A randomized controlled trial of radiofrequency ablation and surgical resection in the treatment of small hepatocellular carcinoma. J Hepatol. 2012 Oct;57(4): 794-802.

10 Liu H, Wang ZG, Fu SY, Li AJ, Pan ZY, Zhou WP, et al. Randomized clinical trial of chemoembolization plus radiofrequency ablation versus partial hepatectomy for hepatocellular carcinoma within the Milan criteria. Br J Surg. 2016 Mar;103(4):34856. 
11 Gerunda GE, Neri D, Merenda R, Barbazza F, Zangrandi F, Meduri F, et al. Role of transarterial chemoembolization before liver resection for hepatocarcinoma. Liver transplantation : official publication of the American association for the study of liver diseases. Liver Transplant. 2000 Sep;6(5):619-26.

12 Oken MM, Creech RH, Tormey DC, Horton J, Davis TE, McFadden ET, et al. Toxicity and response criteria of the Eastern cooperative oncology group. Am J Clin Oncol. 1982 Dec; 5(6):649-55.

13 Kudo M, Kubo S, Takayasu K, Sakamoto M, Tanaka M, Ikai I, et al. Response evaluation criteria in cancer of the liver (RECICL) proposed by the liver cancer study group of Japan (2009 revised version). Hepatol Res. 2010 Jul; 40(7):686-92.

14 Kawaguchi Y, Hasegawa K, Hagiwara Y, De Bellis M, Famularo S, Panettieri E, et al. Effect of diameter and number of hepatocellular carcinomas on survival after resection, transarterial chemoembolization, and ablation. Am J Gastroenterol. 2021.
15 Minagawa M, Makuuchi M, Takayama T, Ohtomo K. Selection criteria for hepatectomy in patients with hepatocellular carcinoma and portal vein tumor thrombus. Ann Surg. 2001 Mar;233(3):379-84.

16 Ni JY, Xu LF, Sun HL, Zhou JX, Chen YT, Luo $\mathrm{JH}$. Percutaneous ablation therapy versus surgical resection in the treatment for early-stage hepatocellular carcinoma: a meta-analysis of 21,494 patients. J Cancer Res Clin Oncol. 2013 Dec;139(12):2021-33.

17 Qi X, Tang Y, An D, Bai M, Shi X, Wang J, et al. Radiofrequency ablation versus hepatic resection for small hepatocellular carcinoma: a meta-analysis of randomized controlled trials. J Clin Gastroenterol. 2014 May;48(5): 450-7.

18 Hasegawa K, Kokudo N, Imamura H, Matsuyama Y, Aoki T, Minagawa M, et al. Prognostic impact of anatomic resection for hepatocellular carcinoma. Ann Surg. 2005 Aug; 242(2):252-9.

19 Takayama T, Makuuchi M, Hirohashi S, Sakamoto M, Okazaki N, Takayasu K, et al. Malignant transformation of adenomatous hyperplasia to hepatocellular carcinoma. Lancet. 1990 Nov 10;336(8724):1150-3.
20 Kubo S, Nishiguchi S, Hirohashi K, Shuto T, Kuroki T, Minamitani S, et al. Clinicopathological criteria for multicentricity of hepatocellular carcinoma and risk factors for such carcinogenesis. Jpn J Cancer Res. 1998 Apr; 89(4):419-26.

21 Ahmed M, Solbiati L, Brace CL, Breen DJ, Callstrom MR, Charboneau JW, et al. Imageguided tumor ablation: standardization of terminology and reporting criteria: a 10-year update. Radiology. 2014 Nov;25(11):1691$705 \mathrm{e} 4$.

22 Lam VW, Ng KK, Chok KS, Cheung TT, Yuen J, Tung $\mathrm{H}$, et al. Risk factors and prognostic factors of local recurrence after radiofrequency ablation of hepatocellular carcinoma. J Am College Surg. 2008 Jul;207(1):20-9.

23 Liu B, Long J, Wang W, Huang T, Xie X, Chen $S$, et al. Predictive factors of treatment outcomes after percutaneous ablation of hepatocellular carcinoma in the caudate lobe: a retrospective study. BMC cancer. 2019 Jul 16; 19(1):699. 